Portland State University

PDXScholar

7-1-2011

\title{
Striation-Based Beamforming for Estimating the Waveguide Invariant with Passive Sonar
}

\author{
Lisa M. Zurk \\ Portland State University, zurkl@pdx.edu \\ Daniel Rouseff \\ University of Washington - Seattle Campus
}

Follow this and additional works at: https://pdxscholar.library.pdx.edu/ece_fac

Part of the Electrical and Computer Engineering Commons

Let us know how access to this document benefits you.

\section{Citation Details}

Rouseff, D., \& Zurk, L. M. (2011). Striation-based beamforming for estimating the waveguide invariant with passive sonar. The Journal of the Acoustical Society of America, 130(2), EL76-EL81.

This Article is brought to you for free and open access. It has been accepted for inclusion in Electrical and Computer Engineering Faculty Publications and Presentations by an authorized administrator of PDXScholar. Please contact us if we can make this document more accessible: pdxscholar@pdx.edu. 


\title{
Striation-based beamforming for estimating the waveguide invariant with passive sonar
}

\author{
Daniel Rouseff \\ Applied Physics Laboratory, University of Washington, 1013 NE 40th Street, Seattle, Washington 98105 \\ rouseff@apl.washington.edu \\ Lisa M. Zurk \\ Department of Electrical and Computer Engineering, Portland State University, Portland, Oregon 97201 \\ zurkl@pdx.edu
}

\begin{abstract}
The waveguide invariant summarizes the pattern of constructive and destructive interference between acoustic modes propagating in the ocean waveguide. For many sonar signal-processing schemes, it is essential to know the correct numerical value for the waveguide invariant. While conventional beamforming can estimate the ratio between the waveguide invariant and the range to the source, it cannot unambiguously separate the two terms. In the present work, striationbased beamforming is developed. It is shown that the striation-based beamformer can be used to produce an estimate for the waveguide invariant that is independent of the range. Simulation results are presented.
\end{abstract}

(c) 2011 Acoustical Society of America

PACS numbers: 43.60.Fg, 43.30.Cq, 43.30.Wi [JFL]

Date Received: January 31, 2011 Date Accepted: June 3, 2011

\section{Introduction}

Consider an underwater passive sonar scenario where a horizontal array measures the acoustic field produced by a distant source. Mapped as a function of frequency $\omega$ and range $r$, the observed acoustic intensity $I$ often exhibits striations. These striations, alternating bands of high and low intensity, result from constructive and destructive interference between the propagating acoustic modes supported by the ocean waveguide. Chuprov $^{1}$ developed a simple formula for the slope $d \omega / d r$ of the striations:

$$
\frac{d \omega}{d r}=\frac{-\partial I / \partial r}{\partial I / \partial \omega}=\omega \beta / r
$$

where $\beta$ is the so-called waveguide invariant. Typically, one would know the frequency and measure the slope allowing the $\beta / r$ ratio to be calculated.

While the ratio between the waveguide invariant and the range may be readily determined, isolating the range from the numerical value for $\beta$ is more problematic. In practice, one might simply assume that $\beta$ is known and then calculate $r$ from the $\beta / r$ ratio. The canonical value in shallow water is $\beta=1$, but this choice may prove far too coarse. If both the source and array are below the thermocline, both simulations ${ }^{2}$ and experiments ${ }^{3,4}$ have shown that $\beta=1.3$ or 1.4 is more appropriate. Erroneously assuming $\beta=1$ when it is actually $\beta=1.3$ translates directly into a $30 \%$ error in the estimated value for the range.

In the present work, a method is proposed for isolating the waveguide invariant and producing an estimate for its numerical value that is independent of the range. The method relies on twice beamforming the acoustic pressure measured on a horizontal array due to a distant source. The pressure field is first beamformed conventionally at a single frequency to achieve a focus at the correct bearing of the source. The field is then beamformed again but with each point on the array evaluated at a slightly 
different frequency. The frequency offset is selected based on the observed slope of the intensity striations. It is shown that the focus will shift from what is observed with conventional beamforming and that the extent of the shift gives a measure of $\beta$ that is independent of $r$. The method also gives an indirect coarse estimate of the source's depth.

To begin the development, assume a point source at $\mathbf{r}_{0}=\left(r_{0} \cos \phi_{0}\right.$, $\left.r_{0} \sin \phi_{0}, z_{0}\right)$ in the ocean waveguide. The resulting field is measured on a horizontal line array of length $L$ oriented along the $y$-axis, $-L / 2<y<L / 2$, at depth $z_{a}$. Assuming a range-independent environment and an $\exp (-i \omega t)$ convention, the measured complex field is expanded in normal modes as

$$
p(y, \omega)=\sum_{m}\left(\xi_{m} r\right)^{-1 / 2} \Psi_{m}\left(z_{0}\right) \Psi_{m}\left(z_{a}\right) \exp \left(i \xi_{m} r\right),
$$

where each mode $\Psi_{m}$ has corresponding horizontal wavenumber $\xi_{m}$ and certain unimportant scaling factors are neglected. It is assumed that the source is coherent over the narrow frequency band of interest. The range $r$ to a point on the array is

$$
\begin{aligned}
r & =\left[\left(r_{0} \cos \phi_{0}\right)^{2}+\left(y-r_{0} \sin \phi_{0}\right)^{2}\right]^{1 / 2} \\
& \approx r_{0}-y \sin \phi_{0}+y^{2} /\left(2 r_{0}\right)
\end{aligned}
$$

to the term second order in $y$ that expresses wavefront curvature.

Since $I=|p|^{2}$, the slope of the observed intensity follows from Eqs. (1)-(3):

$$
\frac{d \omega}{d y}=\frac{d \omega}{d r} \frac{d r}{d y} \approx-\omega\left(\beta / r_{0}\right) \sin \phi_{0},
$$

where the distant source is assumed not too near broadside, $\phi_{0}=0$, so the wavefront curvature term can be neglected. Solving the separable differential equation yields

$$
\ln \left(\omega / \omega_{0}\right)=-\left(\beta / r_{0}\right) \sin \left(\phi_{0}\right) y,
$$

that may be approximated for frequencies near the center frequency $\omega_{0}$ as

$$
\omega=\omega_{0}-S y, \text { where } S=\omega_{0}\left(\beta / r_{0}\right) \sin \phi_{0} .
$$

Equation (6) says that the level curves of constant intensity as observed along the array will map onto straight lines of slope $S$. Consequently, incoherent processing using only the intensity is sufficient to estimate $S$. The numerical value for $S$ might be calculated by spectral methods, as originally suggested by Chuprov, ${ }^{1}$ or perhaps by using Radon transforms. ${ }^{5}$ Regardless of the specific method used to do the calculation, the remainder of this report assumes that the value for $S$ is known.

Having first processed the measured field incoherently to deduce $S$, it is now processed coherently by beamforming. Define the beamformer output in look direction $\phi$ as

$$
B(\phi) \equiv|b(\phi)|^{2} \text { with } b(\phi)=L^{-1} \int_{-L / 2}^{L / 2} p(y, \omega) w^{*}(y, \omega ; \phi) d y .
$$

For a conventional Bartlett beamformer, the weights are

$$
w^{*}(y, \omega ; \phi)=\exp \left[i\left(\omega / c_{0}\right) \sin (\phi) y\right],
$$

where $c_{0}$ is the reference sound speed. Substituting Eqs. (2) and (8) into (7) and rearranging terms yields 


$$
b(\phi)=\sum_{m} C_{m} D_{m}(\omega ; \phi) .
$$

The terms slowly varying in $y$ and $\omega$ are lumped into $C_{m}$. The rapidly varying phase terms are included in $D_{m}$ :

$$
D_{m}(\omega ; \phi)=L^{-1} \int_{-L / 2}^{L / 2} \exp \left[i \xi_{m}(\omega) r+i\left(\omega / c_{0}\right) \sin (\phi) y\right] d y,
$$

where the frequency dependence of the horizontal wavenumber $\xi_{m}$ has been made explicit.

In conventional beamforming, the frequency is fixed at some constant value, say, $\omega=\omega_{0}$. In the present work, a striation-based beamformer is considered where different points along the array are evaluated at different frequencies. The frequency varies linearly across the array based on the observed slope $S$ of the intensity striations. To maintain generality, let the frequency across the array be

$$
\omega=\omega_{0}-\alpha S y,
$$

where $\alpha$ is an adjustable parameter. For conventional beamforming, $\alpha=0$ and the frequency is constant across the array. For beamforming along the striations, $\alpha=1$; see Eq. (6). The frequency-dependent modal wavenumber across the array can be approximated by a Taylor's series expansion. To first order,

$$
\begin{aligned}
\xi_{m}\left(\omega_{0}-\alpha S y\right) & \approx \xi_{m}\left(\omega_{0}\right)+\left(\partial \xi_{m} / \partial \omega\right)(-\alpha S y) \\
& =\omega_{0} / v_{m}-\left(\alpha S / u_{m}\right) y,
\end{aligned}
$$

where $v_{m}$ and $u_{m}$ are the phase and group velocities, respectively, for mode $m$ at $\omega=\omega_{0}$. The expansion in Eq. (12) should retain the quadratic term to be strictly consistent with the expansion in Eq. (3). The quadratic term in expansions like Eq. (12) is usually neglected; see the discussion in Ref. 6 for the limitation associated with this approximation.

Combining the above equations yields for the striation-based beamformer

$$
D_{m}\left(\omega_{0}-\alpha S y ; \phi\right)=L^{-1} \int_{-L / 2}^{L / 2} \exp \left[i\left(\theta_{0}+\theta_{1} y+\theta_{2} y^{2}\right)\right] d y,
$$

where terms have been grouped in like order of $y$ with coefficients

$$
\begin{gathered}
\theta_{0}=\left(\omega_{0} / v_{m}\right) r_{0}, \\
\theta_{1}=\left(\omega_{0} / c_{0}\right) \sin (\phi)-\left(\omega_{0} / v_{m}\right) \sin \left(\phi_{0}\right)-\alpha S r_{0} / u_{m} \\
=\left(\omega_{0} / c_{0}\right) \sin (\phi)-\left(\omega_{0} / v_{m}\right) \sin \left(\phi_{0}\right)\left[1+\alpha \beta\left(v_{m} / u_{m}\right)\right], \\
\theta_{2}=\left(\omega_{0} / v_{m}\right) /\left(2 r_{0}\right)+\left(\alpha S / u_{m}\right)\left[\sin \left(\phi_{0}\right)-\left(u_{m} / c_{0}\right) \sin (\phi)\right] .
\end{gathered}
$$

It is instructive to consider the linear coefficient $\theta_{1}$ in greater detail. In transitioning between the two forms of Eq. (15), the definition of the striation slope $S$, Eq. (6), has been applied. Since $S$ is proportional to the ratio $\beta / r_{0}$, the product $S r_{0}$ appearing in Eq. (15) is independent of $r_{0}$. The key point is that $\theta_{1}$ therefore depends on the waveguide invariant but is independent of range; $\beta$ has been isolated from $r_{0}$ by striationbased beamforming.

Equation (13) can be evaluated exactly in terms of Fresnel integrals. ${ }^{7}$ To gain greater insight into the result, it is useful to assume that the source is in the far field of 
the receiving array. In the far field, the quadratic term in Eq. (13) is neglected and the integral reduces to

$$
D_{m}\left(\omega_{0}-\alpha S y ; \phi\right)=e^{i \theta_{0}} \operatorname{sinc}\left(\theta_{1} L / 2\right),
$$

where $\operatorname{sinc}(x) \equiv \sin (x) / x$. The magnitude is a maximum when $\theta_{1}=0$ implying focusing when the beamformer look direction $\phi$ satisfies

$$
\begin{aligned}
\sin (\phi) & =\left(c_{0} / v_{m}\right) \sin \left(\phi_{0}\right)\left[1+\alpha \beta\left(v_{m} / u_{m}\right)\right] \\
& \approx \sin \left(\phi_{0}\right)(1+\alpha \beta),
\end{aligned}
$$

where the approximate form ignores the small differences between $c_{0}$ and the phase and group velocities to yield a result that is independent of the mode index.

Equations (6) and (18) suggest a three step processing strategy for estimating the waveguide invariant. First, process the field incoherently to estimate the slope $S$ of the intensity striations. Second, beamform conventionally with $\alpha=0$. The beamformer will focus at the true bearing of the source, $\phi=\phi_{0}$. Finally, perform striation-based beamforming with $\alpha \neq 0$. The focus will shift to some new $\phi \neq \phi_{0}$ but, since the true $\phi_{0}$ has already been estimated, the location of the new focus can be used in Eq. (18) to solve for $\beta$. The value for the waveguide invariant is thereby estimated without knowing the range or depth of the source.

The numerical value for the adjustable parameter $\alpha$ should be selected with some care. The choice $\alpha=1$ is attractive because it means a strong signal across the array without fades. This should improve performance in a noisy environment. When the source is at a bearing too far from array broadside, however, the choice $\alpha=1$ will fail to produce a focus. For example, no beamformer look direction $\phi$ will satisfy Eq. (18) if $\alpha=\beta=1.0$ and $\left|\phi_{0}\right|>30^{\circ}$. If conventional beamforming $(\alpha=0)$ reveals a source far removed from broadside, the striation-based beamformer should use a value for $\alpha$ that ensures that Eq. (18) will have a solution. In addition, $\alpha$ must be selected so that the total frequency shift across the array, $\alpha S L$, is within the coherence bandwidth of the source.

A specific numerical simulation serves to illustrate the striation-based beamforming concept. Consider an environment typical of the East China Sea as observed during a 2001 experiment. ${ }^{8}$ The $105 \mathrm{~m}$ deep water column has a $40 \mathrm{~m}$ deep surface mixed layer above a sharp thermocline. The sound speed contrast between the surface mixed layer and bottom of the water column is $12 \mathrm{~m} / \mathrm{s}$. Assume a 128 element horizontal receiving array with $0.5 \mathrm{~m}$ spacing $(L=63.5 \mathrm{~m})$ positioned at depth $z_{a}=60 \mathrm{~m}$. The source is positioned at range $r_{0}=5 \mathrm{~km}$ and bearing relative to array broadside $\phi_{0}$ $=20^{\circ}$. The initial source depth is $z_{0}=60 \mathrm{~m}$ so that both the source and the receiving array are positioned below the part of the thermocline with a sharp gradient. With both the source and the receiving array below the main thermocline, the low-order acoustic modes that have an upper turning depth below the sea surface will contribute strongly to the acoustic field. In such a scenario, previous results ${ }^{2-4}$ show that $\beta>1.0$ might be expected.

Figure 1 shows the simulated intensity as calculated along the array over a 50 $\mathrm{Hz}$ band. The calculation was made using a normal mode code and the plot has $20 \mathrm{~dB}$ dynamic range. Striations are plainly evident. A plausible estimate for the slope of the striations is $S=0.089 \mathrm{~Hz} / \mathrm{m}$ implying a $S L=5.7 \mathrm{~Hz}$ frequency shift over the length of the array. Superimposed on the image are lines corresponding to $\alpha=0$ and $\alpha=1$ using reference frequency $\omega_{0} / 2 \pi=1 \mathrm{kHz}$ in Eq. (11).

A discrete Bartlett beamformer was implemented and Fig. 2 shows the results. Beamforming conventionally $(\alpha=0)$, the figure shows a focus at look direction $\phi=\phi_{0}=20^{\circ}$ as expected. The plot is normalized so the conventional $B\left(\phi_{0}\right)=1.0$. The field was then beamformed along the striation $(\alpha=1)$ with the same normalization applied and the result plotted. As expected, the output at the focus is higher for the 


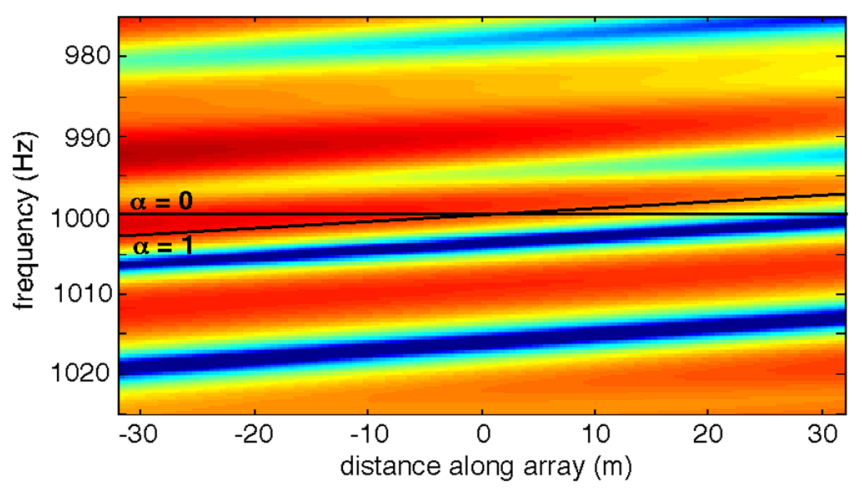

Fig. 1. (Color online) Acoustic intensity observed across horizontal array as function of frequency. Superimposed lines $\alpha=0$ and $\alpha=1$ denote trajectories for conventional and striation-based beamforming, respectively. Dynamic range is $20 \mathrm{~dB}$. See text for simulation details.

striation-based beamformer. More importantly, the location of the focus has shifted to $\phi=52^{\circ}$. Using $\phi=52^{\circ}$ and $\phi_{0}=20^{\circ}$ in Eq. (18) yields a value for the waveguide invariant $\beta=1.3$, a value consistent with experimental results ${ }^{3,4}$ when both source and receiver are below the main thermocline.

The simulation was repeated with all the same parameters except the source depth was changed to $z_{0}=10 \mathrm{~m}$. With the source in the surface mixed layer, the loworder modes that cause $\beta>1.0$ are not excited. Striations are again evident, but with a shallower slope than is observed in Fig. 1; $S=0.069 \mathrm{~Hz} / \mathrm{m}$ is a reasonable value. The conventional and striation-based beamformers focus at $\phi=\phi_{0}=20^{\circ}$ and $\phi=43^{\circ}$, respectively. Using these angles in Eq. (18) yields $\beta=1.0$ for the shallow source.

Because the striation-based beamformer is able to quantify $\beta$, it also provides a coarse estimate of the source depth, at least to the extent that it can distinguish between shallow and deep sources. A caveat is that the present analysis has assumed a range-independent environment. Range dependence from such environmental factors as internal waves will cause the acoustic modes to couple ${ }^{7}$ and make source depth estimation more problematic.

To summarize, while conventional beamforming can determine a source's bearing and the $\beta / r_{0}$ ratio, it is unable to separate the waveguide invariant $\beta$ from the range $r_{0}$. Striation-based beamforming breaks the $\beta / r_{0}$ ambiguity and provides an independent estimate for the waveguide invariant.

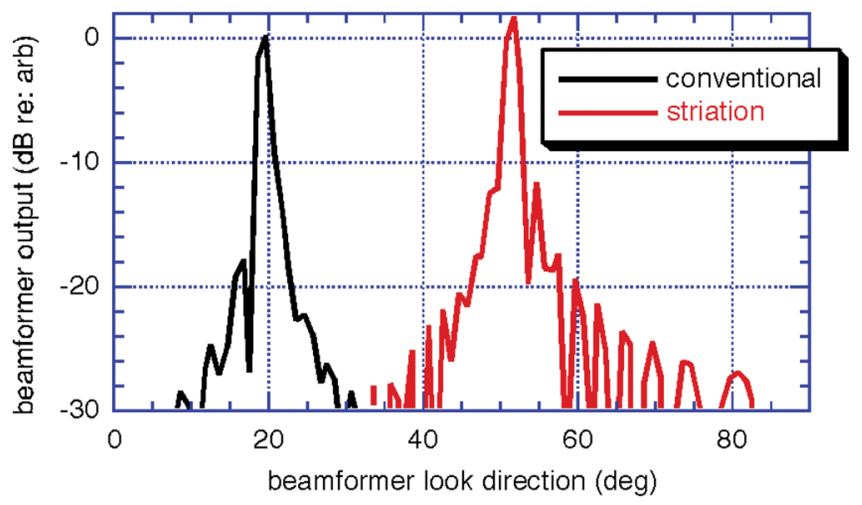

Fig. 2. (Color online) Beamforming results. True source at bearing $\phi_{0}=20^{\circ}$. Shift in location of focus from conventional $(\alpha=0)$ to striation-based $(\alpha=1)$ beamforming can be used to estimate the waveguide invariant using Eq. (18). See text for details. 


\section{Acknowledgments}

This work was supported by the Office of Naval Research.

\section{References and links}

${ }^{1}$ S. D. Chuprov, "Interference structure of a sound field in a layered ocean," in Ocean Acoustics: Current

State, edited by L. M. Brekhovskikh and I. B. Andreevoi (Nauka, Moscow, 1982), pp. 71-91.

${ }^{2}$ D. Rouseff and R. C. Spindel, "Modeling the Waveguide Invariant as a Distribution," in Ocean Acoustic Interference Phenomena and Signal Processing, edited by W. A. Kuperman and G. L. D'Spain (AIP, New York, 2002), pp. 137-148.

${ }^{3}$ W. S. Hodgkiss, H. C. Song, W. A. Kuperman, T. Akal, C. Ferla and D. R. Jackson, “A long-range and variable focus phase-conjugation experiment in shallow water," J. Acoust. Soc. Am. 105, 1597-1604 (1999).

${ }^{4}$ J. E. Quijano, L. M. Zurk, and D. Rouseff, "Demonstration of the invariance principle for monstatic active sonar," J. Acoust. Soc. Am. 123, 1329-1337 (2008).

${ }^{5}$ M. Born and E. Wolf, Principles of Optics, 7th ed. (Cambridge University Press, Cambridge, 1999), pp. 219-227.

${ }^{6}$ J. A. Stratton, Electromagnetic Theory (McGraw-Hill, New York, 1941), pp. 330-340.

${ }^{7}$ Handbook of Mathematical Functions, edited by M. Abramowtiz and I. A. Stegun (U. S. Govt. Printing Office, Washington, D.C., 1964), p. 304.

${ }^{8}$ D. Rouseff and D. Tang, "Internal wave effects on the ambient noise notch in the East China Sea: model/ data comparison,” J. Acoust. Soc. Am. 120, 1284-1294 (2006). 